\title{
Establishment of a xylarium
}

\author{
D. Lamichhane ${ }^{1}$
}

$\mathbf{T}$ he Xylarium is mainly intended to display the wood collections for scientific research, teaching, environmental education and other xylarium programs. This activity is useful for forest utilization research and education. Nevertheless, it is not a new activity of the Department because wood samples of some 32 species are already existed in the library. Most of these species are of lowland or Terai origin. Therefore, with a view to increase diversity of wood samples, 21 more species specimens were collected from midhills of the country. The study area includes forest and farmland of Kaski and Syangja districts. The specimen collection was carried out during Feb.April, 2008 and the wood treatment and labeling was done consequently. The dimension of the specimens is: length: 8", breadth: 4", thickness: 1". Each specimen has a label of its local name, scientific name and main uses.

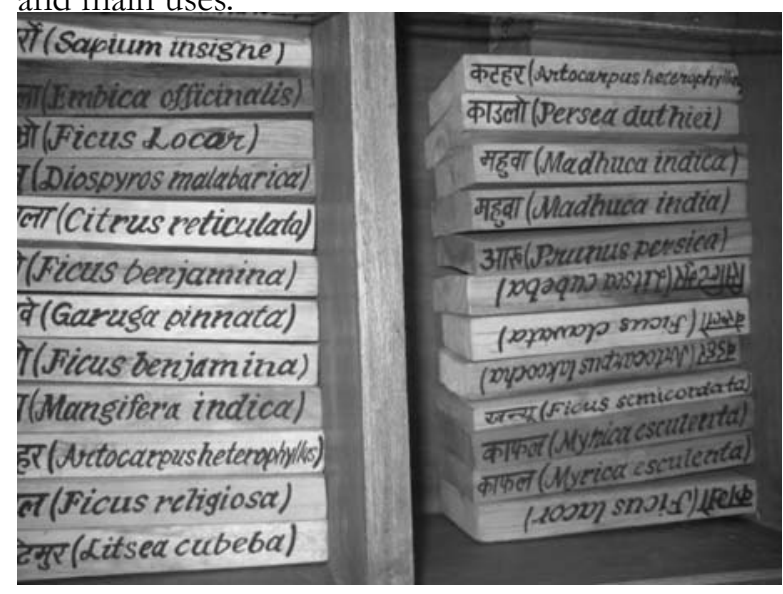

Photo: Xylarium

List of the species of newly collected specimens

\begin{tabular}{lll}
\hline SN & Local Name & Scientific Name \\
\hline 1 & Amala & Emblica officinalis \\
2 & Amp & Mangifera indica \\
3 & Aru & Prunus persica \\
4 & Babul & Acacia arabica \\
5 & Badahar & Artocarpus lakoocha \\
6 & Bar & Ficus benghalensis \\
7 & Berulo & Ficus clavata \\
8 & Dabdabe & Garuga pinnata \\
\hline
\end{tabular}

\begin{tabular}{lll}
\hline 9 & Kafal & Myrica esculenta \\
10 & Katahar & Artocarpus heterophyllus \\
11 & Kaulo & Persea duthiei \\
12 & Kavro & Ficus lacor \\
13 & Khanyu & Ficus semicordata \\
14 & Khirro & Sapium insigne \\
15 & Mahuwa & Madhuca indica \\
16 & Phaledo & Erythrina arborescens \\
17 & Pipal & Ficus religiosa \\
18 & Sami & Ficus benjamina \\
19 & Siltimur & Litsea cubeba \\
20 & Suntala & Citrus reticulata \\
21 & Teju & Diospyros malabarica \\
\hline
\end{tabular}

The other species already collected and displayed in the central forest library of DFRS are as follows: Amaro (Spondias pinnata), Asana (Terminalia tomentosa), Banjh (Q. lanata), Bhudkul (Hymendictyon excelsum), Bot dhayero (Lagerstroemia parviflora), Champ (Michelia champaca), Chilaune (Schima wallichii), Dhale katus (Castanopsis indica), Gobre salla (Pinus excelsa), Gutel (Trewia nudiflora), Harro (Terminalia chebula), Jamun (Eugenia jambolana), Jhingat (Lannea grandis), Karma (Adina cordifolia), Khasru (Q. semecarpifolia), Khayer (Acacia catechu), Khote sallo (Pinus roxburghii), Koiralo (Baubinia variegata), Lakuri (Fraxinus floribunda), Lapsi (Choerospondias axillaris), Musure katus (Castanopsis tribuloides), Okhar (Juglans regia), Paiyu (Prunus cerasoides), Phalant (Q. glauca), Sadan (Ougenia dalbergoides), Sal (Shorea robusta), Satisal (Dalbergia latifolia), Seto siris (Albizia procera), Simal (Bomax ceiba), Sisoo (Dalbergia sissoo), Tuni (Cedrela toona), and Uttis (Alnus nepalensis).

Thus, the Xylarium consists of the specimens of altogether 53 woody species of Terai and Midhills physiographic regions of Nepal and has been displayed in DFRS. It will be useful for researchers, scientists, students, farmers, traders and other concerned stakeholders.

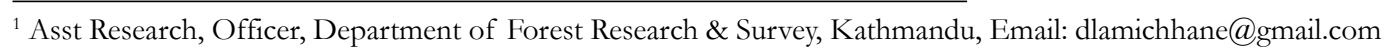

